\title{
A survey of TREM2 antibodies reveals neuronal but not microglial staining in formalin-fixed paraffin- embedded postmortem Alzheimer's brain tissues
}

\author{
Jun-ichi Satoh*1, Natsuki Kawana', Yuji Yamamoto', Tsuyoshi Ishida², Yuko Saito³ and Kunimasa Arima ${ }^{4}$
}

\begin{abstract}
Introduction
Recently, a significant association was identified between a rare rs75932628- $\mathrm{T}$ variant encoding $\mathrm{R} 47 \mathrm{H}$ in the TREM2 gene and an increased risk for Alzheimer's disease (AD) $[1,2]$. TREM2 forms a signaling complex with DAP12 expressed on monocytes/macrophages, dendritic cells, osteoclasts, and microglia. Loss-of-function mutations in either DAP12 or TREM2 are identified in patients with Nasu-Hakola disease, a rare autosomal recessive disorder characterized by presenile dementia and multifocal bone cysts. TREM2 binds to bacterial components and Hsp60, although endogenous ligands remain unknown. $\mathrm{R} 47 \mathrm{H}$ located within the extracellular immunoglobulin-like domain of TREM2 possibly causes a reduced affinity of TREM2 for putative ligands. Knockdown of TREM2 on mouse microglia inhibits phagocytosis of apoptotic neurons and stimulates production of tumor necrosis factor-alpha (TNF $\alpha$ ) and interleukin-1beta (IL-1 $\beta$ ), suggesting a pivotal role for TREM2 in the clearance of damaged neural tissues to resolve damageinduced inflammation. TREM2 is upregulated in amyloid plaque-associated microglia in mouse models of $\mathrm{AD}$, suggesting an active role for TREM2 in the clearance of deposited amyloid- $\beta$ by microglia. However, the precise pathological role of TREM2-expressing microglia in $\mathrm{AD}$ brains remains unknown.
\end{abstract}

\section{Findings}

By immunohistochemistry (IHC), we studied the expression of TREM2 in serial sections of formalin-fixed paraffin-embedded tissues of the frontal cortex and the hippocampus derived from five patients with $\mathrm{AD}$, four patients with amyotrophic lateral sclerosis, and four neurologically normal control subjects. Written informed consent was obtained from all of the cases. The ethics

*Correspondence: satoj@my-pharm.ac.jp

'Department of Bioinformatics and Molecular Neuropathology, Meiji

Pharmaceutical University, 2-522-1 Noshio, Kiyose, Tokyo 204-8588, Japan

Full list of author information is available at the end of the article committee of the corresponding institutions approved the present study. The tissue sections were treated for antigen retrieval in $10 \mathrm{mM}$ citrate sodium buffer, $\mathrm{pH}$ 6.0, at $110^{\circ} \mathrm{C}$ for 15 minutes in a temperature-controlled pressure chamber (Biocare Medical, Concord, CA, USA). First, we evaluated the specificity of commercially available anti-TREM2 antibodies, such as HPA010917 (Sigma-Aldrich, St. Louis, MO, USA), AF1828 (R\&D Systems, Inc., Minneapolis, MN, USA), 2 B5 (Novus Biologicals, Inc., Littleton, CO, USA), bs-2723R (Bioss, Woburn, MA, USA), PA5-18763 (Thermo Fisher Scientific Inc., Waltham, MA, USA), ab69405 (Abcam, Cambridge, UK), and ab85851 (Abcam) by Western blot of recombinant human TREM2 protein expressed in HEK293 cells. Only HPA010917, AF1828, and 2B5, but not others, reacted with TREM2 on the blot (Figure 1). Next, we studied TREM2 expression in spleen, bone marrow, and the brain by IHC with HPA010917, AF1828, and 2B5. Only HPA010917 labeled monocytes/macrophages, dendritic cells, and osteoclasts (Figure 2a,b). Thus, only HPA010917 satisfied the specificity and the reactivity to TREM2 both in vitro and in vivo. By IHC with HPA010917, we found an accumulation of Iba1- and DA12-immunoreactive microglia, but Iba1-, HLA-DR-, CD68-, or DAP12-immunoreactive microglia did not express TREM2 in any of the cases examined (Figures 2c,d and 3a-f). In contrast, TREM2 was identified in small subpopulations of intravascular monocytes/macrophages and neurons and was totally absorbed by preincubation with recombinant protein (Figures $2 c-f$ and $3 b$ ).

Here we found that Iba1-immunoreactive microglia do not express TREM2 in AD and control brains. Previous studies by IHC with uncharacterized antibodies showed that microglia express TREM2 in the mouse and human cerebral cortex, where it is located in the Golgi complex [3]. Although the possibility exists that neuronal staining of TREM2 reflects immunoreactivity of cross-reactive proteins, the validation requires the development of additional antibodies highly specific for distinct epitopes of TREM2. Not all microglia in the mouse brain express TREM2, and its levels are reduced in cultured microglia 

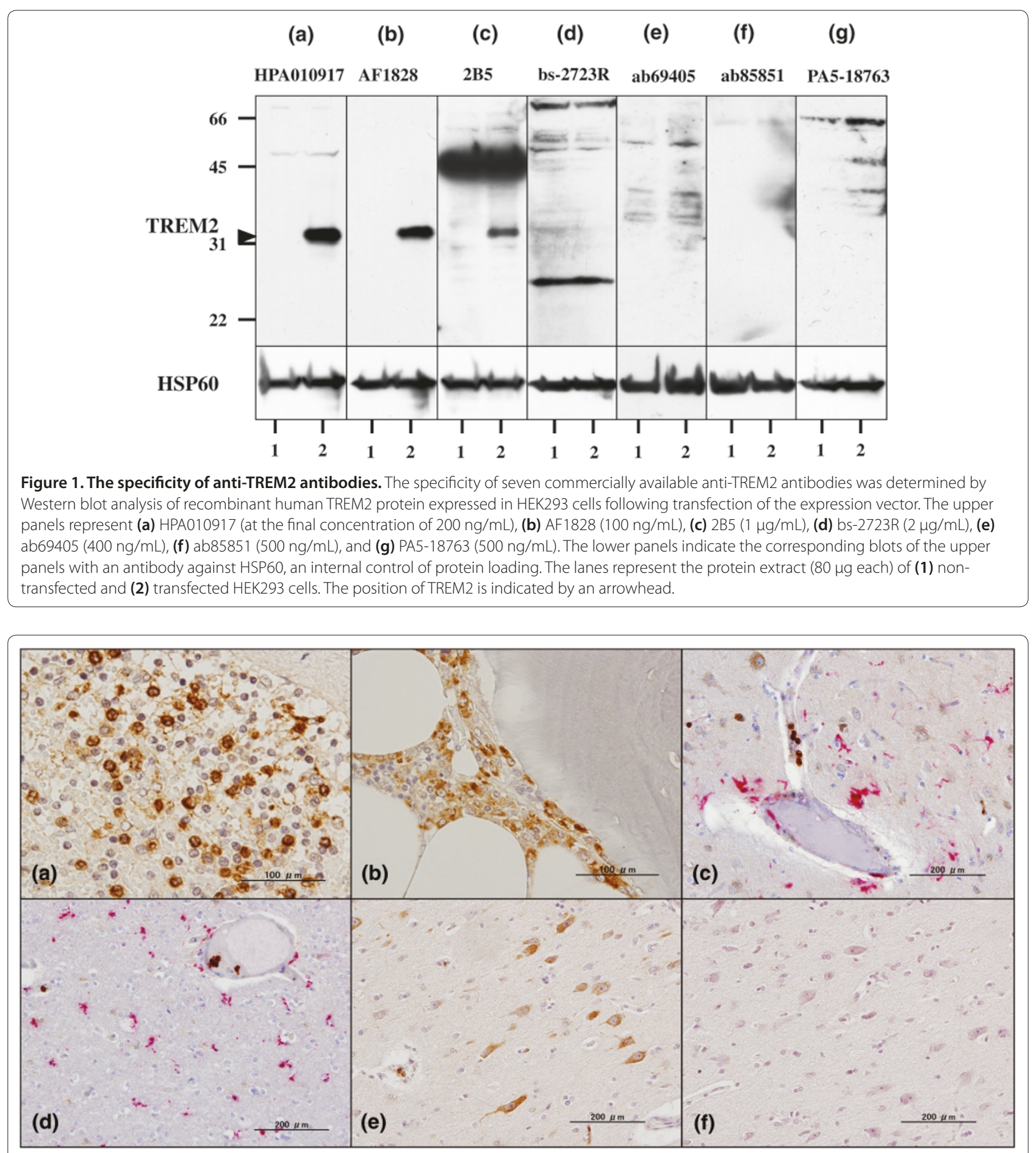

Figure 2. TREM2 immunohistochemistry of spleen, bone marrow, and brain tissues. The panels represent (a) normal control, spleen, HPA010917; (b) normal control, bone marrow (L1), HPA010917; (c) Alzheimer's disease (AD), the frontal cortex, HPA010917 (brown) and HLADR (red); (d) AD, the frontal cortex, HPA010917 (brown) and CD68 (red); (e) AD, the frontal cortex, HPA010917; and (f) the same region as (e), HPA010917 absorbed by preincubation with recombinant TREM2 protein.

by exposure to lipopolysaccharide. Microglial phenotype is changeable from a proinflammatory/neurotoxic (M1) trait to an anti-inflammatory/neuroprotective (M2) trait in response to disturbances of brain homeostasis, suggesting that TREM2 expression on microglia is a transient event in vivo, depending on their microenvironment. 


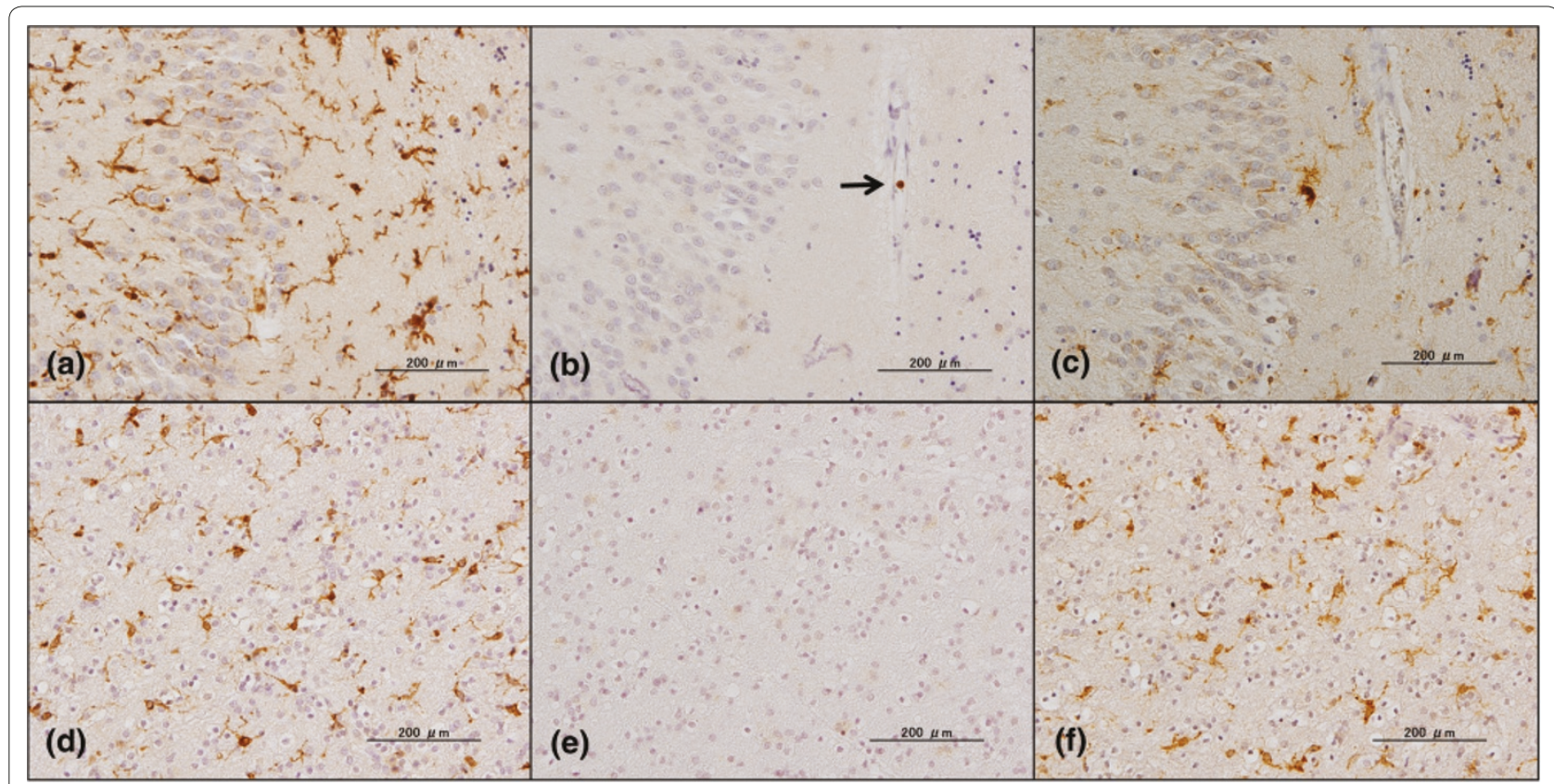

Figure 3. TREM2 immunohistochemistry of Alzheimer's disease (AD) brains. The panels represent serial sections of (a) AD, the hippocampus, Iba1; (b) AD, the same region as (a), HPA010917; (c) AD, the same region as (a), DAP12; (d) AD, the frontal white matter, Iba1; (e) AD, the same region as (d), HPA010917; and (f) AD, the same region as (d), DAP12. In (b), an arrow indicates an intravascular TREM2-expressing monocyte/macrophage.

\section{Abbreviations}

AD, Alzheimer's disease; $I H C$, immunohistochemistry.

\section{Competing interests}

The authors declare that they have no competing interests.

\section{Acknowledgments}

This work was supported by grants from the Genome Research Center (GRC) Project and the Grant-in-Aid (C25430054), of the Ministry of Education, Culture, Sports, Science and Technology (MEXT), Japan.

\section{Author details}

'Department of Bioinformatics and Molecular Neuropathology, Meiji Pharmaceutical University, 2-522-1 Noshio, Kiyose, Tokyo 204-8588, Japan. 2Department of Pathology and Laboratory Medicine, Kohnodai Hospital, National Center for Global Health and Medicine, 1-7-1 Kohnodai, Ichikawa, Chiba 272-8516, Japan. ${ }^{3}$ Department of Laboratory Medicine, National Center Hospital, NCNP, 4-1-1 Ogawahigashi, Kodaira, Tokyo 187-8551, Japan. ${ }^{4}$ Department of Psychiatry, National Center Hospital, NCNP, 4-1-1 Ogawahigashi, Kodaira, Tokyo 187-8551, Japan.

Published: 8 July 2013

\section{References}

1. Jonsson T, Stefansson H, Steinberg S, Jonsdottir I, Jonsson PV, Snaedal J, Bjornsson S, Huttenlocher J, Levey AI, Lah JJ, Rujescu D, Hampel H, Giegling I, Andreassen OA, Engedal K, Ulstein I, Djurovic S, Ibrahim-Verbaas C, Hofman A, Ikram MA, van Duijn CM, Thorsteinsdottir U, Kong A, Stefansson K: Variant of TREM2 associated with the risk of Alzheimer's disease. N Engl J Med 2013, 368:107-116.

2. Guerreiro R, Wojtas A, Bras J, Carrasquillo M, Rogaeva E, Majounie E, Cruchaga C, Sassi C, Kauwe JS, Younkin S, Hazrati L, Collinge J, Pocock J, Lashley T, Williams J, Lambert JC, Amouyel P, Goate A, Rademakers R, Morgan K, Powell J, St George-Hyslop P, Singleton A, Hardy J; Alzheimer Genetic Analysis Group: TREM2 variants in Alzheimer's disease. N Engl J Med 2013, 368:117-127.

3. Sessa G, Podini P, Mariani M, Meroni A, Spreafico R, Sinigaglia F, Colonna M, Panina P, Meldolesi J: Distribution and signaling of TREM2/DAP12, the receptor system mutated in human polycystic lipomembraneous osteodysplasia with sclerosing leukoencephalopathy dementia. Eur J Neurosci 2004, 20:2617-2628.

\section{doi:10.1186/alzrt184}

Cite this article as: Satoh J-i, et al: A survey of TREM2 antibodies reveals neuronal but not microglial staining in formalin-fixed paraffin-embedded postmortem Alzheimer's brain tissues. Alzheimer's Research \& Therapy 2013, 5:30. 